\title{
Demographics, management and outcome of females and males with acute respiratory distress syndrome in the LUNG SAFE prospective cohort study
}

\author{
Bairbre A. McNicholas ${ }^{1,2,7,10}$, Fabiana Madotto 3,10 , Tài Pham (10), 4,6 , \\ Emanuele Rezoagli ${ }^{1,7}$, Claire H. Masterson ${ }^{1}$, Shahd Horie ${ }^{1}$, Giacomo Bellani ${ }^{8,9}$, \\ Laurent Brochard ${ }^{4,5,6}$ and John G. Laffey (1) $^{1,7}$ on behalf of the LUNG SAFE \\ Investigators and the ESICM Trials Group
}

@ERSpublications

Shorter females with ARDS were less likely to receive lower tidal volume ventilation than shorter males, while mortality rates were higher in females with confirmed severe ARDS. Better ventilatory management may improve outcomes in females with ARDS. http://bit.ly/2JIsUBz

Cite this article as: McNicholas BA, Madotto F, Pham T, et al. Demographics, management and outcome of females and males with acute respiratory distress syndrome in the LUNG SAFE prospective cohort study. Eur Respir J 2019; 54: 1900609 [https://doi.org/10.1183/13993003.00609-2019].

\section{ABSTRACT}

Rationale: We wished to determine the influence of sex on the management and outcomes in acute respiratory distress syndrome (ARDS) patients in the Large Observational Study to Understand the Global Impact of Severe Acute Respiratory Failure (LUNG SAFE).

Methods: We assessed the effect of sex on mortality, intensive care unit and hospital length of stay, and duration of invasive mechanical ventilation (IMV) in patients with ARDS who underwent IMV, adjusting for plausible clinical and geographic confounders.

Findings: Of 2377 patients with ARDS, 905 (38\%) were female and 1472 (62\%) were male. There were no sex differences in clinician recognition of ARDS or critical illness severity profile. Females received higher tidal volumes $\left(8.2 \pm 2.1\right.$ versus $\left.7.2 \pm 1.6 \mathrm{~mL} \cdot \mathrm{kg}^{-1} ; \mathrm{p}<0.0001\right)$ and higher plateau and driving pressures compared with males. Lower tidal volume ventilation was received by $50 \%$ of females compared with $74 \%$ of males $(p<0.0001)$. In shorter patients (height $\leqslant 1.69 \mathrm{~m})$, females were significantly less likely to receive lower tidal volumes. Surviving females had a shorter duration of IMV and reduced length of stay compared with males. Overall hospital mortality was similar in females (40.2\%) versus males (40.2\%). However, female sex was associated with higher mortality in patients with severe confirmed ARDS (OR for sex (male versus female) $0.35,95 \%$ CI $0.14-0.83$ ).

Conclusions: Shorter females with ARDS are less likely to receive lower tidal volume ventilation, while females with severe confirmed ARDS have a higher mortality risk. These data highlight the need for better ventilatory management in females to improve their outcomes from ARDS. 


\section{Introduction}

Differences in the clinical management and outcomes of females versus males are well described [1]. The reasons underlying these differences are complex, with biological, organisational, case-mix, ethnicity, socioeconomic and local therapeutic traditions having an influence [2]. Differences related to age profile and disease severity and/or complexity may also play a role [3-5]. For example, females hospitalised with coronary artery disease are less likely to undergo invasive diagnostic and therapeutic intervention despite similar rates of presentation with acute myocardial infarction [1], but these differences may be related to older age at presentation. In females presenting with haemorrhagic stroke, the lower intervention rates is partially explained by more complex disease and older age at presentation [5].

In the critically ill, the impact of sex is less well understood. Sex may affect access to critical care, with females less likely to be admitted to the intensive care unit (ICU) [6]. However, once in the ICU there have been few studies indicating sex bias in the provision of care $[3,6]$. In a prospective study of ICU admissions in Austria, while females had greater illness severity, males were independently more likely to receive invasive procedures, although outcomes were not different by sex [6]. Sex-based biological differences may influence the development and/or management of acute respiratory distress syndrome (ARDS) [7-9]. HAN et al. [10] demonstrated that shorter patients (i.e. predominantly females) with severe sepsis-related acute lung injury received lung protective ventilation less frequently, a finding confirmed in an analysis of ARDS network trials [11]. Height is frequently inaccurately estimated, particularly in shorter patients [12], further increasing risk in females, given their shorter stature. Sex-specific hormonal differences can influence inflammation and immunological function $[13,14]$, which may impact on the risk of developing ARDS. The impact of sex on outcomes from ARDS is less clear [15-17].

We wished to address these issues in a secondary analysis of the Large Observational Study to Understand the Global Impact of Severe Acute Respiratory Failure (LUNG SAFE), a global multicentre cohort study [7]. Our primary objective was to determine the impact of sex on outcomes from ARDS. Secondary objectives were to assess differences in clinician recognition, patient management and progression of ARDS by sex.

\section{Methods}

Study design, patients and data collection

The detailed methods and protocol for LUNG SAFE have been published elsewhere [7]. In brief, LUNG SAFE was an international, multicentre, prospective cohort study, conducted during 4 consecutive weeks in the winter of 2014 in a convenience sample of 459 ICUs from 50 countries across six continents [7]. The study, funded by the European Society of Intensive Care Medicine (ESICM), was endorsed by multiple national societies/networks (supplementary appendix S1). National coordinators and site investigators were responsible for obtaining ethics committee approval, and for ensuring data integrity and validity (supplementary appendix S1). Further details are available in the supplementary material.

LUNG SAFE enrolled patients with acute hypoxaemic respiratory failure (AHRF) admitted to a study ICU who underwent invasive or noninvasive ventilation. Exclusion criteria were age $<16$ years or inability to obtain informed consent (where required). Patients were classified as having ARDS if they fulfilled all of the Berlin criteria [7]. We restricted subsequent analyses to patients that fulfilled ARDS criteria (93\%) within $48 \mathrm{~h}$ of the onset of $\mathrm{AHRF}$ and who received invasive mechanical ventilation (IMV) (supplementary figure E1).

\section{Data definitions}

Our data definitions have been previously reported [7, 18, 19]. For the purposes of this analysis, sex assignment was made by the site investigators at the time of data entry. Lower tidal volume (LTV) ventilation was defined as a tidal volume $\leqslant 8 \mathrm{~mL} \cdot \mathrm{kg}^{-1}$ ideal body weight (IBW). In patients in whom

Affiliations: ${ }^{1}$ Regenerative Medicine Institute (REMEDI), CÚRAM Centre for Research in Medical Devices, School of Medicine, National University of Ireland Galway, Galway, Ireland. ${ }^{2}$ Nephrology Services, Galway University Hospitals, SAOLTA University Healthcare Group, Galway, Ireland. ${ }^{3}$ Research Centre on Public Health, School of Medicine and Surgery, University of Milano-Bicocca, Monza, Italy. ${ }^{4}$ Keenan Research Centre for Biomedical Science, St Michael's Hospital, Toronto, ON, Canada. ${ }^{5}$ Dept of Critical Care Medicine, St Michael's Hospital, Toronto, ON, Canada. Interdepartmental Division of Critical Care Medicine, University of Toronto, Toronto, ON, Canada. ${ }^{7}$ Dept of Anaesthesia and Intensive Care Medicine, Galway University Hospitals, SAOLTA University Healthcare Group, Galway, Ireland. ${ }^{8}$ School of Medicine and Surgery, University of MilanBicocca, Monza, Italy. ${ }^{9}$ Dept of Emergency and Intensive Care, San Gerardo Hospital, Monza, Italy. ${ }^{10}$ These authors are joint first authors.

Correspondence: John G. Laffey, Anaesthesia and Intensive Care Medicine, School of Medicine, National University of Ireland Galway, University Road, Galway, H91 TK33, Ireland. E-mail: john.laffey@nuigalway.ie 
plateau pressure was measured, lung protective ventilation (LPV) was defined as tidal volume $\leqslant 8 \mathrm{~mL} \cdot \mathrm{kg}^{-1}$ IBW and plateau pressure $\leqslant 30 \mathrm{cmH}_{2} \mathrm{O}$. From the variables originally collected we also derived dynamic compliance and body mass index (BMI). We used the threshold of $1.69 \mathrm{~m}$ (median height value) to classify shorter versus taller patients. Gross domestic product (GDP) per person was obtained through the World Bank database that gathers time series data for all countries on a variety of socioeconomic topics. GDP was used to define three major geoeconomic groupings: high-income countries in Europe, high-income countries in the rest of the world and middle-income countries (http://databank.worldbank.org/data/home. aspx). Duration of IMV was calculated as the number of days between the date of intubation and the date of extubation in the ICU (or death, if the patient died under IMV). Similarly, invasive ventilator-free days were calculated as the number of days from weaning from IMV to day 28, while patients who died before weaning were considered to have a ventilator-free day value of 0 . Length of stay (LOS) in the ICU and hospital was evaluated as the number of days between the date of admission and the date of discharge from the ICU and hospital, respectively. Survival was evaluated at ICU and hospital discharge or at day 90, whichever occurred first. Because we previously observed a significant association between the presence of ARDS at day 2 and outcomes [20], ARDS severity was reclassified ("resolved" versus "confirmed" ARDS) on day 2 using the Berlin criteria.

\section{Data management and statistical analyses}

Descriptive statistics were reported for the study population stratified according to sex, and included proportions for categorical variables and mean with standard deviation or median (interquartile range) for continuous variables. No assumptions were made for missing data, which were rare. Comparisons between groups were performed using the Chi-squared test (or Fisher's exact test) for discrete variables and the t-test (or Wilcoxon-Mann-Whitney test) for continuous variables. The Shapiro-Wilk test was used to assess normality in data distribution.

The Kaplan-Meier approach was applied to assess the probability of discontinuing IMV in the ICU, and the probability of hospital survival and of being discharged alive during hospital stay. When assessing the probability of discontinuing IMV in the ICU, patients that weaned from IMV after 28 days in the ICU were considered as censored at day 28 . When assessing the probability of being discharged alive from hospital, patients that died before day 90 were considered as censored at date of death, while patients discharged after day 90 were considered as censored at day 90 . The log-rank test was used to compare curves between the female and male populations.

To evaluate the existence of a possible effect of sex on mortality, LTV ventilation during the first day of ARDS, LOS and duration of IMV adjusting for all plausible confounders, we applied generalised linear mixed models with random intercept, taking into account the correlation among patients within the same ICU of enrolment. Patients who died before ICU discharge were removed from the analysis of LOS and duration of IMV. In detail, the logistic-link function and binomial distribution of outcome were used to analyse mortality and LTV ventilation, while the log-link function and Poisson distribution were used for LOS and duration of IMV. In the first case, results were reported as odds ratio with $95 \%$ confidence interval, while in the second as incidence rate ratio with $95 \%$ confidence interval. As some ICUs had few observations to support the normal assumption, the bootstrap method was used (1000 samples randomly extracted) to estimate the model parameters.

Predictors used in the multivariable models were detected through the stepwise regression approach that combines forward and backward selection methods in an iterative procedure (significance level of 0.05 both for entry and retention). Potential independent predictors were: patient characteristics at baseline (age, sex, BMI, geoeconomic area), chronic disease (chronic obstructive pulmonary disease (COPD), diabetes mellitus, immuno-incompetence, cardiac failure, renal failure, liver failure), presence of ARDS risk factors, ICU characteristics (number of beds, proportion of ICU beds in hospital, number of beds per physician and per nurse, academic ICU), clinical parameters measured on day 2 from ARDS onset (total respiratory rate, tidal volume, presence of controlled ventilation, positive end-expiratory pressure (PEEP), standardised minute ventilation, ARDS severity, dynamic compliance, partial pressure of carbon dioxide $\left(P_{\mathrm{aCO}_{2}}\right), \mathrm{pH}$, nonpulmonary Sequential Organ Failure Assessment (SOFA) score, presence of adjunctive measures performed during 2 day from ARDS onset). Moreover, when sex was identified as a statistical significant predictor, we also evaluated its interaction with other selected predictors.

All p-values were two-sided, with $\mathrm{p}<0.05$ considered as statistically significant. Statistical analyses were performed with R version 3.5.2 (www.R-project.org) and SAS version 9.4 (SAS Institute, Cary, NC, USA).

\section{Results}

Of the 12906 patients enrolled in LUNG SAFE, 4499 developed criteria for AHRF: 1716 (38.1\%) females and 2783 (61.9\%) males (supplementary figure E1). Identical proportions of females and males were 
observed in the study population of 2377 patients who developed ARDS within 2 days from AHRF onset and were managed with IMV (supplementary figure E1). The proportions of males and females aged $\geqslant 50$ years were also unchanged in the study population (table 1 ).

In the study population, female patients $(n=905)$ were shorter and had a higher BMI, and a greater frequency of immuno-incompetence (i.e. steroid use, cancer or haematological malignancies), while male patients $(n=1472)$ had a higher frequency of COPD and chronic renal failure (table 1). There were differences in risk factor profile, with gastric aspiration, major trauma, pulmonary contusion and

TABLE 1 Characteristics of the 2377 invasively ventilated female and male patients with acute respiratory distress syndrome (ARDS)

\begin{tabular}{|c|c|c|c|}
\hline & Females & Males & p-value ${ }^{\#}$ \\
\hline Patients & $905(38.1)$ & $1472(61.9)$ & \\
\hline Patients $>\mathbf{5 0}$ years old & 665 (37.2) & $1123(62.8)$ & \\
\hline Age years & $60.1 \pm 17.3$ & $60.8 \pm 16.5$ & 0.4057 \\
\hline Height m & $1.60 \pm 0.08$ & $1.73 \pm 0.08$ & $<0.0001$ \\
\hline$B M I \mathrm{~kg} \cdot \mathrm{m}^{-2}$ & $28.8 \pm 9.2$ & $26.8 \pm 8.4$ & $<0.001$ \\
\hline \multicolumn{4}{|l|}{ Chronic disease } \\
\hline COPD & $157(17.3)$ & $315(21.4)$ & 0.0162 \\
\hline Diabetes mellitus & 195 (21.5) & $320(21.7)$ & 0.9121 \\
\hline Immuno-incompetence (all types) & 205 (22.7) & $280(19.0)$ & 0.0330 \\
\hline Chronic cardiac failure & $82(9.1)$ & $142(9.6)$ & 0.6349 \\
\hline Chronic renal failure & $71(7.8)$ & $153(10.4)$ & 0.0389 \\
\hline Chronic liver failure & 32 (3.5) & $71(4.8)$ & 0.1344 \\
\hline \multicolumn{4}{|l|}{ Specific risk factor for ARDS ${ }^{\pi}$} \\
\hline Pneumonia & $541(59.8)$ & 844 (57.3) & 0.2410 \\
\hline Nonpulmonary sepsis & 170 (18.8) & 243 (16.5) & 0.1549 \\
\hline Aspiration of gastric contents & $116(12.8)$ & $256(17.4)$ & 0.0029 \\
\hline Noncardiogenic shock & $84(9.3)$ & $115(7.8)$ & 0.2092 \\
\hline Major trauma & $22(2.4)$ & $85(5.8)$ & 0.0001 \\
\hline Blood transfusion & $44(4.9)$ & $59(4.0)$ & 0.3209 \\
\hline Pulmonary contusion & $18(2.0)$ & $62(4.2)$ & 0.0035 \\
\hline Inhalation injury & $17(1.9)$ & $51(3.5)$ & 0.0243 \\
\hline Drug overdose & $14(1.5)$ & $35(2.4)$ & 0.1663 \\
\hline Pulmonary vasculitis & $2(0.2)$ & $7(0.5)$ & 0.4966 \\
\hline Severe burns & $4(0.4)$ & $4(0.3)$ & 0.4890 \\
\hline Drowning & $0(0.0)$ & $2(0.1)$ & 0.5283 \\
\hline Pancreatitis & $13(1.4)$ & $37(2.5)$ & 0.0756 \\
\hline Other & 30 (3.3) & $39(2.6)$ & 0.2481 \\
\hline Risk factor for ARDS & & & 0.2625 \\
\hline Only pulmonary risk factors & $511(56.6)$ & 843 (57.3) & \\
\hline Only nonpulmonary risk factors & $196(21.7)$ & $286(19.4)$ & \\
\hline Both & 126 (13.9) & $239(16.2)$ & \\
\hline No risk factor & $72(8.0)$ & $104(7.1)$ & \\
\hline \multicolumn{4}{|l|}{ Type of admission } \\
\hline Medical & $692(76.5)$ & $1073(72.9)$ & 0.0532 \\
\hline Post-operative (elective) & $55(6.1)$ & $88(6.0)$ & 0.9214 \\
\hline Surgical & $136(15.0)$ & $230(15.6)$ & 0.6952 \\
\hline Trauma & $22(2.4)$ & $81(5.5)$ & 0.0004 \\
\hline \multicolumn{4}{|l|}{ Clinician recognition of ARDS } \\
\hline At baseline & $300(33.1)$ & $486(33.0)$ & 0.9467 \\
\hline During ICU stay & $592(65.4)$ & 938 (63.7) & 0.4031 \\
\hline \multicolumn{4}{|l|}{ ICU characteristics } \\
\hline Beds & $17.0(11.0-24.0)$ & $17.0(11.0-25.0)$ & 0.6169 \\
\hline Proportion of beds in hospital & $2.5(1.5-4.5)$ & $2.6(1.5-4.4)$ & 0.4057 \\
\hline Bed per physician & $5.0(2.7-10.0)$ & $4.8(2.7-9.4)$ & 0.3759 \\
\hline Bed per nurse & $1.4(1.0-2.0)$ & $1.4(1.0-2.0)$ & 0.9712 \\
\hline Academic & $687(78.4)$ & $1083(76.1)$ & 0.1897 \\
\hline
\end{tabular}

Data are presented as $\mathrm{n}(\%)$, mean \pm SD or median (interquartile range), unless otherwise stated. BMI: body mass index; COPD: chronic obstructive pulmonary disease; ICU: intensive care unit. ${ }^{\#}$ : comparison of male versus female patients; ${ }^{\text {ๆ: }}$ total is $>100 \%$, since patients could have more than one risk factor. 
inhalation injury each less frequent in females (table 1). There was no difference in rates of clinician recognition of ARDS by sex.

There were no differences by sex with regard to the severity profile of ARDS or overall severity of illness as defined by SOFA score on day 1 or 2 of ARDS (table 2 and supplementary table E1). No statistically significant differences were observed between the sexes in the progression of ARDS severity, whether in mild $(\mathrm{p}=0.2191)$, moderate $(\mathrm{p}=0.3575)$ or severe $(\mathrm{p}=0.1613)$ patients on day 2 (supplementary figure $\mathrm{E} 2)$. There were no sex differences in inspired oxygen use, arterial oxygen partial pressure/inspiratory oxygen fraction ratio or oxygen saturation on day 1 or 2 .

The management of IMV differed by sex (figure 1, table 2 and supplementary table E1). Tidal volumes were higher in females, both in those that received controlled and assisted mechanical ventilation. Peak, plateau and driving pressures were higher, and standardised minute ventilation was higher, in females on days 1 and 2 compared with males (figure $1 \mathrm{a}$ and $\mathrm{b}$, table 2, and supplementary table E2). In patients in whom plateau pressure was measured, more male patients received LPV ( $75 \%$ versus $51 \%$; $<<0.0001)$ (figure 1c and d). In contrast, there were no differences in PEEP levels used. $P_{\mathrm{aCO}_{2}}$ was lower and minute volume higher in females on day 1 of ARDS (table 2), and a greater proportion of females were hypocapnic, while more males were hypercapnic (table 2).

Tidal volume sizes increased at lower height quintiles in both sexes (figure 1e). At the lowest quintile (height $\leqslant 1.60 \mathrm{~m}$ ) where $>80 \%$ were female, tidal volume was significantly higher in females (figure 1e). Tidal volumes were higher in females at each quintile of actual body weight (supplementary figure E3a). LOESS (locally weighted smoothing) regression demonstrated a clear relationship between lower height and higher tidal volume in females and males (supplementary figure E3b). At similar dynamic compliance, females received higher tidal volumes compared with males (supplementary figure E3c). Females continued to receive larger tidal volumes over each day of follow-up to day 14 (supplementary figure E3c and supplementary table E3). More females received adjunctive measures $(\mathrm{p}=0.0322)$, with extracorporeal membrane oxygenation (ECMO) and inhaled vasodilators used more frequently in females compared with males (table 2).

Multivariable models were constructed to examine factors associated with the use of LTV ventilation (supplementary table E4 and figure 1f). Factors including higher $P_{\mathrm{aCO}}$, controlled ventilation, higher $\mathrm{pH}$ and geoeconomic area were associated with LTV use (supplementary table E6). In shorter patients (i.e. height less than or equal to the median value of $1.69 \mathrm{~m}$ ), male sex was independently associated with use of LTV ventilation (figure 1f).

There were differences in the recovery profile from ARDS between the sexes. Female patients had a shorter duration of IMV and reduced length of ICU and hospital stay compared with males (table 3). In surviving patients, female patients had more invasive ventilator-free days in the ICU, a shorter duration of IMV and reduced length of ICU and hospital stay compared with males (table 3). The overall probability of being discharged alive by day 90 was higher for female patients (figure $2 \mathrm{a}$ ).

Overall ICU and hospital mortality rates were identical for both females and males (table 3). KaplanMeier analyses demonstrated no differences in the probability of discontinuing IMV or of hospital mortality during follow-up between the sexes (figure $2 b$ and $c$ ). There were no differences in limitation of life-sustaining measures such as withdrawal or withholding of support between females and males (table 3).

When patients were stratified by severity of ARDS on day 2, ICU mortality ( $64 \%$ versus 46\%; $\mathrm{p}=0.0066$ ) and hospital mortality ( $68 \%$ versus $50 \%$; $\mathrm{p}=0.0068$ ) were significantly higher for females who had severe "confirmed" ARDS compared with males (supplementary figure E4 and supplementary table E2). KaplanMeier analyses of hospital survival in female and male patients stratified by ARDS severity (at day 2) demonstrated a significantly lower probability of survival in females with severe confirmed ARDS (figure 3).

After adjusting for all potential confounders and considering the correlation among patients within the same ICU of enrolment, female sex was independently associated with reduced length of IMV and shorter ICU and hospital stay in survivors (figure $4 \mathrm{a}$ and supplementary table E5). Female sex was associated with increased ICU and hospital mortality in patients with severe confirmed ARDS (figure $4 \mathrm{~b}$ and supplementary table E5). Subsequent analysis of males and females with severe confirmed ARDS revealed no major differences in demographics or illness severity, but there were significant differences in ventilatory management between the sexes (supplementary table E6).

While there were geoeconomic area differences in the use of LTV ventilation (supplementary table E4) and in overall outcomes of ARDS (supplementary tables E5 and E7) as previously reported [21], geoeconomic area did not modify the relationships between sex and outcomes. 
TABLE 2 Ventilatory management and illness severity of the 2377 invasively ventilated female and male patients with acute respiratory distress syndrome (ARDS)

\begin{tabular}{|c|c|c|c|}
\hline & Females & Males & p-value $\#$ \\
\hline Subjects & 905 & 1472 & \\
\hline \multicolumn{4}{|l|}{ Invasive ventilation settings at ARDS onset (day 1 ) } \\
\hline Patients undergoing controlled ventilation & $631(70.6)$ & $980(68.1)$ & 0.1995 \\
\hline Set respiratory rate breaths $\min ^{-1}$ & $18.5 \pm 6.1$ & $18.7 \pm 10.0$ & 0.9509 \\
\hline Total respiratory rate breaths $\min ^{-1}$ & $20.6 \pm 6.4$ & $20.9 \pm 9.7$ & 0.7230 \\
\hline \multicolumn{4}{|l|}{ Tidal volume $\mathrm{mL} \cdot \mathrm{kg}^{-1} \mathrm{IBW}$} \\
\hline All patients & $8.2 \pm 2.1$ & $7.2 \pm 1.6$ & $<0.0001$ \\
\hline Patients with controlled ventilation & $8.0 \pm 2.0$ & $7.1 \pm 1.5$ & $<0.0001$ \\
\hline Patients with spontaneous ventilation & $8.6 \pm 2.2$ & $7.5 \pm 1.8$ & $<0.0001$ \\
\hline p-value (control versus spontaneous ventilation) & 0.0005 & 0.0003 & \\
\hline Lower tidal volume & $424(49.6)$ & 1039 (74.2) & $<0.0001$ \\
\hline Set PEEP $\mathrm{cmH}_{2} \mathrm{O}$ & $8.5 \pm 3.4$ & $8.4 \pm 3.3$ & 0.9046 \\
\hline Peak pressure ${ }^{+} \mathrm{cmH}_{2} \mathrm{O}$ & $28.0 \pm 8.6$ & $26.5 \pm 7.9$ & $<0.0001$ \\
\hline Dynamic compliance $\mathrm{mL} \cdot \mathrm{cmH}_{2} \mathrm{O}^{-1}$ & $27.31 \pm 22.83$ & $34.65 \pm 32.61$ & $<0.0001$ \\
\hline Patients in whom $P_{\text {PLAT }}$ was measured & $371(41.0)$ & $583(39.6)$ & 0.5025 \\
\hline$P_{\text {PLAT }} \mathrm{cmH}_{2} \mathrm{O}^{\S}$ & $24.1 \pm 6.0$ & $22.6 \pm 6.1$ & 0.0003 \\
\hline Driving pressure $\mathrm{cmH}_{2} \mathrm{O}^{\S}$ & $15.7 \pm 5.6$ & $14.1 \pm 5.4$ & 0.0001 \\
\hline Standardised minute ventilation $L \cdot \min ^{-1 f}$ & $9.61 \pm 4.4$ & $11.53 \pm 5.1$ & 0.0001 \\
\hline Standardised minute ventilation $\mathrm{L} \cdot \mathrm{min}^{-1} \cdot \mathrm{kg}^{-1} \mathrm{IBW} f$ & $0.18 \pm 0.09$ & $0.17 \pm 0.07$ & $<0.0001$ \\
\hline \multicolumn{4}{|l|}{ Gas exchange (day 1 ) } \\
\hline$P_{\mathrm{aO}_{2}} / F_{\mathrm{IO}_{2}} \mathrm{mmHg}$ & $162.1 \pm 68.7$ & $159.6 \pm 67.5$ & 0.4261 \\
\hline $\mathrm{S}_{\mathrm{pO}_{2}}$ & $96.0(93.0-98.0)$ & $96.0(93.0-98.0)$ & 0.9153 \\
\hline $\mathrm{PaCO}_{2} \mathrm{mmHg}$ & $45.2 \pm 15.5$ & $46.5 \pm 14.6$ & 0.0008 \\
\hline$<35 \mathrm{mmHg}$ & $190(21.3)$ & $244(16.7)$ & 0.0061 \\
\hline $35-45 \mathrm{mmHg}$ & $341(38.1)$ & $535(36.7)$ & 0.4804 \\
\hline$\geqslant 45 \mathrm{mmHg}$ & $363(40.6)$ & $679(46.6)$ & 0.0047 \\
\hline $\mathrm{pH}^{\prime}$ & $7.32 \pm 0.13$ & $7.33 \pm 0.12$ & 0.8145 \\
\hline \multicolumn{4}{|l|}{ Severity profile (day 1 ) } \\
\hline ARDS severity & & & 0.8743 \\
\hline Mild & $275(30.4)$ & $439(29.8)$ & \\
\hline Moderate & $423(46.7)$ & $683(46.4)$ & \\
\hline Severe & $207(22.9)$ & $350(23.8)$ & \\
\hline SOFA score $\# \#$ & $9.9 \pm 4.0$ & $10.1 \pm 4.0$ & 0.1713 \\
\hline Nonpulmonary SOFA score ${ }^{\# \#}$ & $6.7 \pm 4.0$ & $6.9 \pm 3.9$ & 0.2266 \\
\hline $\mathrm{F}_{\mathrm{IO}_{2}}$ & $0.6(0.5-0.9)$ & $0.6(0.5-0.9)$ & 0.7265 \\
\hline \multicolumn{4}{|l|}{ Adjunctive measures (first $48 \mathrm{~h}$ ) } \\
\hline Neuromuscular blockade & 160 (17.7) & $245(16.6)$ & 0.5144 \\
\hline Recruitment manoeuvres & $173(19.1)$ & $251(17.1)$ & 0.2017 \\
\hline Prone positioning & $48(5.3)$ & $65(4.4)$ & 0.3231 \\
\hline ECMO & $36(4.0)$ & $27(1.8)$ & 0.0016 \\
\hline Inhaled vasodilators & $62(6.9)$ & $65(4.4)$ & 0.0104 \\
\hline HFOV & $1(0.1)$ & $5(0.3)$ & 0.4175 \\
\hline None of the above & 578 (63.9) & $1003(68.1)$ & 0.0322 \\
\hline
\end{tabular}

Data are presented as $\mathrm{n}, \mathrm{n}(\%)$, mean \pm SD or median (interquartile range), unless otherwise stated. IBW: ideal body weight; PEEP: positive end-expiratory pressure; $P_{\mathrm{PLAT}}$ : plateau pressure; $P_{\mathrm{aO}_{2}}$ : arterial oxygen partial pressure; $F_{\mathrm{IO}_{2}}$ : inspiratory oxygen fraction; $S_{\mathrm{pO}_{2}}$ : peripheral oxygen saturation; $P_{\mathrm{acO}}$ : partial pressure of carbon dioxide; SOFA: Sequential Organ Failure Assessment; ECMO: extracorporeal membrane oxygenation; HFOV: high-frequency oscillatory ventilation. " : comparison of male versus female patients; П: lower tidal volume was defined as tidal volume $\leqslant 8 \mathrm{~mL} \cdot \mathrm{kg}^{-1}$ IBW; ${ }^{+}$: for peak pressure measurements, patients receiving HFOV or ECMO were excluded; ${ }^{\S}$ : P PLAT and driving pressure values are limited to patients in whom this value was reported, and in whom either an assist control mode was used or, where a mode permitting spontaneous ventilation was used, the set and total respiratory rates were equal (patients receiving HFOV or ECMO were also excluded): ${ }^{f}$ : standardised minute ventilation was calculated as minute ventilation $\times \mathrm{PaCO}_{2} / 40 ;{ }^{\# \#}$ : for all SOFA scores, where data points were missing, this value was omitted and the denominator adjusted accordingly. 

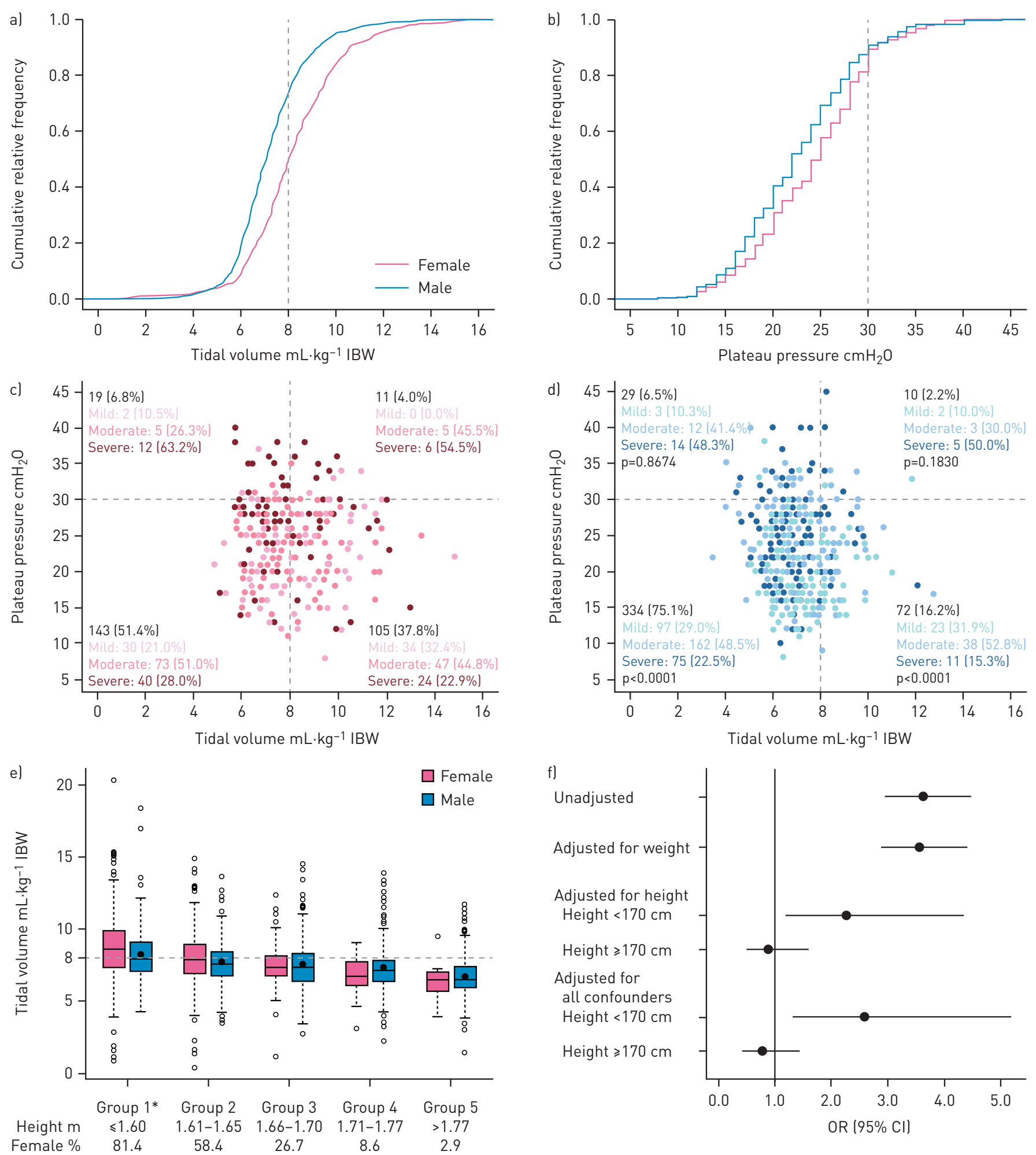

FIGURE 1 Impact of sex on ventilatory parameters at day 1 of acute respiratory distress syndrome (ARDS) in invasively ventilated patients. IBW: ideal body weight; ECMO: extracorporeal membrane oxygenation; HFOV: high-frequency oscillatory ventilation. a, b) Cumulative frequency distribution of a) tidal volume and b) plateau pressure in females $(n=278)$ and males $(n=445)$. $c$, d) Distribution of tidal volume and plateau pressure in c) females ( $n=278$ ) and d) males ( $n=445)$ by ARDS severity at onset. Data in (a)-(c) are limited to patients with available plateau pressure, and in whom either an assist control mode was used or, where a mode permitting spontaneous ventilation was used, the set and total respiratory rates were equal. Patients receiving HFOV or ECMO were also excluded. $p$-values in (d) refer to comparison with female patients. d) Box plot for tidal volume in the female and male populations stratified by quintiles of height in the study population. *: $p<0.05$ for the comparison between females and males. Pearson correlation coefficient between tidal volume and height in females and males is -0.3439 $(p<0.0001)$ and $-0.2855(p<0.0001)$, respectively. The dashed lines in $(a)-(d)$ indicate cut-offs of tidal volume $8 \mathrm{~mL} \cdot \mathrm{kg}^{-1}$ IBW and plateau pressure $30 \mathrm{cmH}_{2} \mathrm{O}$. e) Odds ratios $\left(95 \% \mathrm{Cl}\right.$ ) for males versus females of receiving lower tidal volume ventilation (tidal volume $\leqslant 8 \mathrm{~mL} \cdot \mathrm{kg}^{-1}$ IBW). 
TABLE 3 Outcomes observed during follow-up in the 2377 invasively ventilated female and male patients with acute respiratory distress syndrome

\begin{tabular}{|c|c|c|c|}
\hline & Females & Males & p-value $\#$ \\
\hline Subjects & 905 & 1472 & \\
\hline \multicolumn{4}{|l|}{ Ventilatory support at ICU discharge } \\
\hline All patients & $232(25.64)$ & $351(23.85)$ & 0.3246 \\
\hline Survivors at ICU discharge & $118(20.17)$ & $162(16.98)$ & 0.1154 \\
\hline \multicolumn{4}{|l|}{ Invasive ventilator-free days in ICU" } \\
\hline All patients & $13.0(0.0-23)$ & $10.5(0.0-22.0)$ & 0.1616 \\
\hline Survivors at ICU discharge & $22.0(16.0-25.0)$ & $20.0(13.0-25.0)$ & 0.0134 \\
\hline \multicolumn{4}{|l|}{ Duration of invasive mechanical ventilation in ICU days ${ }^{+}$} \\
\hline All patients & $7.0(4.0-13.0)$ & $9.0(4.0-16.0)$ & 0.0044 \\
\hline Survivors at ICU discharge & $7.0(4.0-13.0)$ & $9.0(4.0-16.0)$ & 0.0124 \\
\hline \multicolumn{4}{|l|}{ Length of stay in ICU days s $^{\mathcal{S}}$} \\
\hline All patients & $9.0(5.0-17.0)$ & $11.0(6.0-20.0)$ & 0.0014 \\
\hline Survivors at ICU discharge & $11.0(6.0-18.0)$ & $12.0(7.0-22.0)$ & 0.0094 \\
\hline \multicolumn{4}{|l|}{ Length of stay in hospital days ${ }^{f}$} \\
\hline All patients & $16.0(8.0-29.0)$ & $18.0(9.0-35.0)$ & 0.0006 \\
\hline Survivors at hospital discharge & $21.0(13.0-36.0)$ & $25.0(14.0-44.0)$ & 0.0012 \\
\hline \multicolumn{4}{|l|}{ Limitation of life-sustaining measures in ICU } \\
\hline All patients & $225(24.9)$ & $353(24.0)$ & 0.6269 \\
\hline Patients who died in ICU after limitation ${ }^{\# \#}$ & $179(79.6)$ & $285(80.7)$ & 0.7279 \\
\hline Time to limitation in ICU ๆา & $50.5 \pm 3.1$ & $54.7 \pm 1.9$ & 0.0834 \\
\hline \multicolumn{4}{|l|}{ ICU mortality } \\
\hline All patients & $320(35.4)$ & $518(35.2)$ & 0.9333 \\
\hline Patients in European high-income countries & $184(39.6)$ & $277(34.7)$ & 0.0837 \\
\hline Patients in non-European high-income countries & 59 (23.3) & $122(30.8)$ & 0.0380 \\
\hline Patients in middle-income countries & $77(41.2)$ & $119(42.8)$ & 0.7272 \\
\hline p-value lamong geoeconomic regions) & $<0.0001$ & 0.0053 & \\
\hline \multicolumn{4}{|l|}{ Hospital mortality ${ }^{++}$} \\
\hline All patients & $362(40.2)$ & $590(40.2)$ & 0.9844 \\
\hline Patients in European high-income countries & $212(45.7)$ & $315(39.5)$ & 0.0309 \\
\hline Patients in non-European high-income countries & $69(27.4)$ & $147(37.3)$ & 0.0091 \\
\hline Patients in middle-income countries & $81(43.8)$ & $128(46.5)$ & 0.5597 \\
\hline $\mathrm{p}$-value (among geoeconomic regions) & $<0.0001$ & 0.0462 & \\
\hline
\end{tabular}

Data are presented as $\mathrm{n}, \mathrm{n}(\%)$, median (interquartile range) or mean $\pm \mathrm{SD}$, unless otherwise stated. ICU: intensive care unit. " : comparison of male versus female patients; ": invasive ventilator-free days were calculated as the number of days from weaning from invasive mechanical ventilation to the date of ICU discharge (patients who died before weaning were considered to have a ventilator-free day value of 0 ); ${ }^{+}$: duration of invasive mechanical ventilation was assessed during ICU stay and was calculated as the number of days between the date of intubation and the date of extubation performed in the ICU; ${ }^{\S}$ : length of stay in the ICU was calculated as the number of days between the date of ICU admission and the date of ICU discharge (or 90 when discharge occurred after 90 days); ${ }^{f}$ : length of stay in hospital was calculated as the number of days between the date of ICU admission and the date of hospital discharge lor 90 when discharge occurred after 90 days); \#\#: percentage was calculated on patients with limitation of

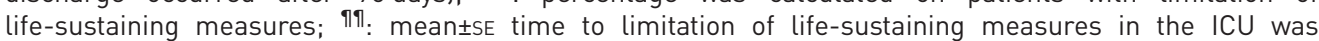
estimated with the Kaplan-Meier approach, considering as censored those patients discharged from the ICU; ${ }^{++}$: vital status at hospital discharge was not evaluable for nine patients (four females and five males).

\section{Discussion}

Important sex-based differences exist in the demographics, management and outcomes of patients with ARDS. There are differences in the demographics and risk factors for ARDS between females and males. Female patients are more likely to receive nonprotective lung ventilation with higher tidal and minute volumes, and higher plateau and driving pressures, compared with males. While this was due in part to their lower height profile, shorter females were more likely to receive LTV ventilation than shorter males. While females had a shorter duration of IMV and a reduced length of ICU and hospital stay, ICU and hospital mortality rates were identical compared with males. Of particular concern is the fact that female sex was independently associated with higher mortality in patients with severe confirmed ARDS.

Demographic differences are consistent with previous studies which found that ARDS occurs more commonly in males than in females $[10,17]$. We found no differences in age or clinician recognition of ARDS. Females with ARDS were shorter and had a higher BMI compared with males. Risk factors such as 

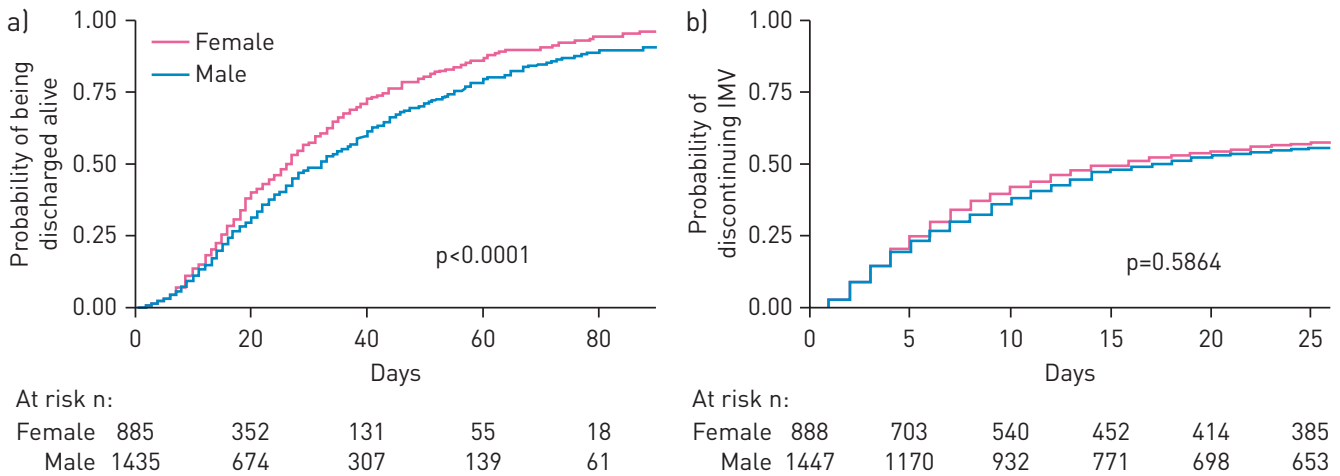

\begin{tabular}{|c|c|c|c|}
\hline Female 885 & 352 & 131 & 55 \\
\hline Male 1435 & 674 & 307 & 139 \\
\hline
\end{tabular}

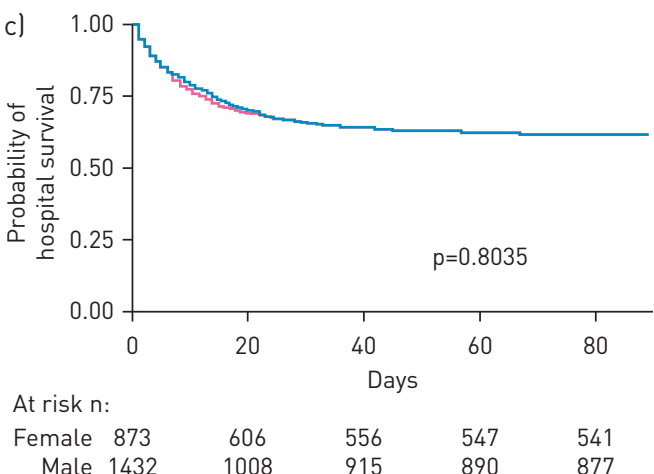

FIGURE 2 Kaplan-Meier curves for main outcomes in acute respiratory distress syndrome (ARDS) patients invasively ventilated, stratified by sex. IMV: invasive mechanical ventilation; ICU: intensive care unit. The number of patients at risk refers to the end of the corresponding day. a) Probability of being discharged alive from hospital. Patients discharged alive/dead after day 90 or dead patients were considered as censored at day 90 or at date of death, respectively. b) Probability of discontinuing IMV in the ICU. Patients with weaning from IMV after 28 days in the ICU were considered as censored at day 28. c) Probability of hospital survival. Patients discharged alive before day 90 were considered alive at day 90 . All p-values are from the log-rank test.

trauma, COPD and chronic renal failure were less common and immunosuppression more common in females, likely reflecting differences in smoking patterns, chronic kidney disease risk and cancer among females and males [22]. Differences in sex hormones, such as oestrogen and testosterone, can influence inflammation and immunological function $[13,14]$, which may impact on the risk of developing ARDS. In pre-pubertal children, where hormone levels should be equally low between males and females, ARDS due to nonseptic causes occurs equally. However, ARDS secondary to sepsis occurs at a greater frequency in male children, suggesting a biological reason for the difference in risk for ARDS where hormonal levels between males and females are equal [14]. In this analysis, in patients aged $>50$ years there was no increase in the proportion of females to males, suggesting that the frequency of ARDS does not increase in females post-menopause.

We next assessed if ventilatory management of ARDS differs between female and male patients with ARDS. A higher proportion of females received nonprotective lung ventilation and a greater proportion were hypocapnic. These differences in ventilation in females persisted out to day 14, suggesting this was sustained over time. If actual body weight rather than IBW is used to calculate the delivered tidal volume, the tidal volume may be inappropriately high [10]. Interestingly, at each quintile of actual body weight, females received significantly higher tidal volumes, while weight was not associated with LTV ventilation use. We found that in shorter patients, both female and male, clinicians were less likely to apply LTV ventilation, confirming and expanding prior findings [10]. While the delivery of higher tidal volumes to females patients has been previously described [11,23], this has been attributed solely to their (shorter) height $[10,11,23]$. Our findings show that female sex is a factor, with shorter females significantly less likely to receive LTV ventilation than similarly sized males.

Mortality in females may have been lower if LPV had been appropriately used. Understanding the barriers to LPV implementation is important $[10,24,25]$. LPV was associated with an $8.8 \%$ reduction in mortality in one study, with another finding that each $1 \mathrm{~mL} \cdot \mathrm{kg}^{-1}$ increase in initial tidal volume above predicted body weight was associated with a $23 \%$ increase in mortality [26]. Overall, females are shorter than males and height measurement errors are magnified in shorter individuals, particularly where height is estimated 

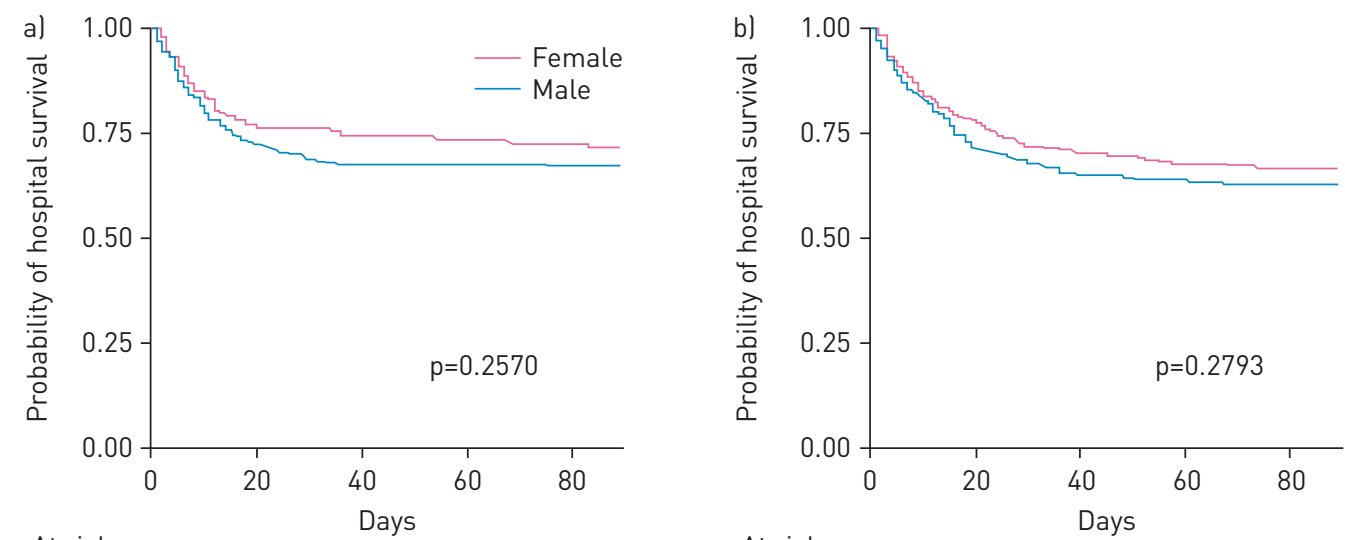

$\begin{array}{lllll}\text { At risk n: } & & & & \\ \text { Female 178 } & 138 & 133 & 131 & 129 \\ \text { Male 321 } & 233 & 218 & 217 & 216\end{array}$

\begin{tabular}{cccccc} 
At risk n: & \multicolumn{5}{c}{ Days } \\
Female & 212 & 166 & 149 & 144 & 142 \\
Male & 330 & 236 & 216 & 212 & 207
\end{tabular}
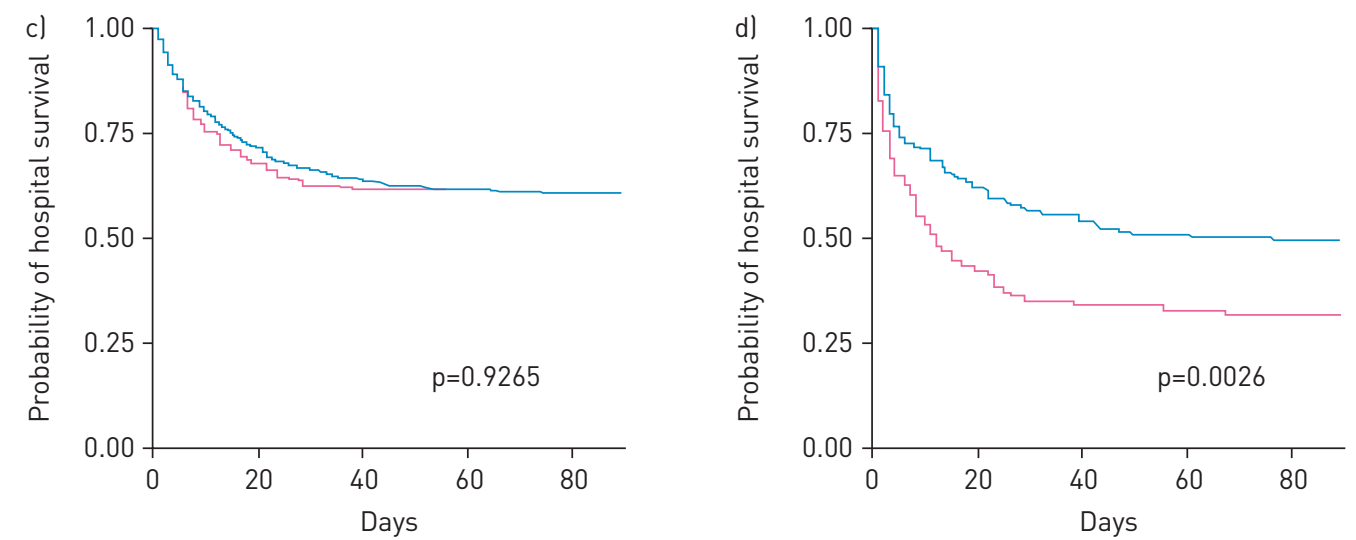

$\begin{array}{lllll}\text { At risk n: } & & & & \\ \text { Female } 300 & 204 & 185 & 185 & 184 \\ \text { Male 525 } & 377 & 337 & 324 & 319\end{array}$

$\begin{array}{lcllll}\text { At risk n: } & & & & \\ \text { Female } & 94 & 40 & 32 & 31 & 30 \\ \text { Male } & 145 & 90 & 78 & 74 & 72\end{array}$

FIGURE 3 Probability of hospital survival in female and male patients stratified by acute respiratory distress syndrome (ARDS) severity at day 2: a) resolved ARDS, b) mild ARDS, c) moderate ARDS and d) severe ARDS. The number of patients at risk refers to the end of the corresponding day. Patients discharged alive before day 90 were considered alive at day 90 . All p-values are from the log-rank test.
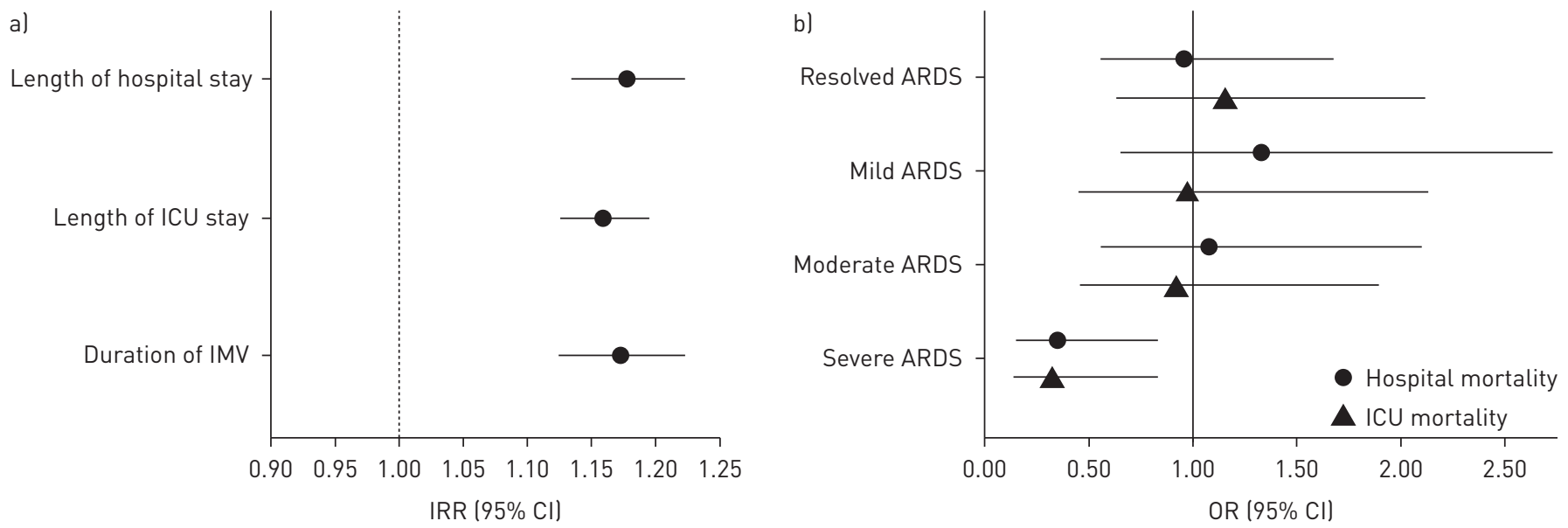

FIGURE 4 Effects of sex on main outcomes in patients with acute respiratory distress syndrome (ARDS). IMV: invasive mechanical ventilation; ICU: intensive care unit; IRR: incidence rate ratio. a) IRR for males versus females for length of ICU stay, length of hospital stay and duration of IMV. b) Odds ratios for males versus females for ICU and hospital mortality by ARDS severity at day 2 (resolved, mild, moderate and severe). 
[12]. In individuals undergoing abdominal surgery $>4 \mathrm{~h}$ duration, female sex, short stature and obesity were associated with use of tidal volumes $>10 \mathrm{~mL} \cdot \mathrm{kg}^{-1}$ [27]. The greater risk of shorter females receiving inappropriately high tidal volumes highlights the need for particular care and attention in calculating and applying LTVs to this cohort.

There were important differences in the recovery profile and outcomes between the sexes. Females with ARDS required shorter ICU stays and had more ventilator-free days. This did not appear to be a "survivor bias" as the finding persisted in surviving patients. Despite this, overall ICU and hospital mortality were identical in females and males with ARDS. Prior studies have reported similar findings, with female patients with critical illnesses including acute lung injury having shorter ICU stays and requiring less resource use $[6,15,17,28,29]$, but with similar mortality rates to their male counterparts. There are pre-clinical and clinical data to suggest that females and males respond differently to inflammation [30] and to mechanical ventilation [17]. In one study, mechanically ventilated females had a faster alveolar fluid clearance rate compared with males [31]. These findings raise the possibility that females may recover from ARDS faster than males, but that this may be nullified by the lower rates of LPV in females. Additional studies are required to further examine these issues.

Our findings regarding outcomes from ARDS provide important additional insights compared with prior studies. Our finding of no overall differences in outcome from ARDS by sex confirms prior findings in a meta-analysis of outcomes from the ARDSnet studies [32]. Other studies have noted worse outcomes for females on mechanical ventilation, but with organ dysfunction explaining most of the differences [33]. We examined outcomes by day 2 severity, as we have previously demonstrated that reclassification at day 2 provides additional insights [20]. The finding that female patients with severe confirmed ARDS had increased mortality is a novel finding and is of concern. Differences in risk factor profile (e.g. greater pneumonia and nonpulmonary sepsis, lower trauma) may explain some of the outcome differences between the sexes, but overall these differences were relatively small. There were relatively little differences in illness severity profiles between males and females with severe confirmed ARDS. However, females with severe ARDS received higher tidal volumes and were exposed to higher airway pressures compared with males with severe ARDS, which is of concern given their worse outcome compared with their male counterparts. In this study, females were not more likely to receive orders relating to limitation of life-sustaining therapies following ICU admission, which is consistent with other studies [34, 35].

As previously reported, differences exist in the management of patients with ARDS based on geoeconomic region [21]. Differences in outcomes related to geoeconomic region were noted, with ICU and hospital mortality lowest in high-income countries. However, geoeconomic difference was not a factor in modifying the relationship between sex and mortality. The proportion of patients with severe ARDS was lower while use of LPV was higher in non-European high-income countries, which might explain the lowest proportion of female patients dying from ARDS in these countries compared with other geoeconomic areas [21]. It is reassuring that no sex-based differences in access to care for patients with ARDS were found in the study.

This study has several limitations related to study design, as have been previously described [7]. Limitations pertaining to this study include the possibility that all females with ARDS were not accounted for, i.e. due to nonadmission and palliation outside of the ICU. Associations of management with worse outcomes in females with severe ARDS were examined only for days 1 and 2 of ARDS. However, early management of ARDS is important to outcome and choosing a longer time-point to analyse would have decreased patient numbers due to ICU discharges or patient death.

In conclusion, higher proportions of shorter females with ARDS receive nonprotective lung ventilation compared with similarly sized males. There appear to be differences in recovery profiles between the sexes, with females requiring shorter ICU stays and more ventilator-free days. Of concern, females with severe confirmed ARDS have a higher mortality compared with their male counterparts. These findings suggest that females with ARDS require particular attention to their ventilatory management in order to optimise their outcomes.

Acknowledgement: A complete list of LUNG SAFE national coordinators, site investigators and national societies endorsing the study can be found in the supplementary material.

Support statement: This work was funded and supported by the European Society of Intensive Care Medicine (ESICM; Brussels, Belgium), by St Michael's Hospital (Toronto, ON, Canada) and by the University of Milan-Bicocca (Monza, Italy). These funders had no role in the design and conduct of the study; management, analysis and interpretation of the data; preparation, review or approval of the manuscript; or decision to submit the manuscript for publication. Funding information for this article has been deposited with the Crossref Funder Registry. 
Author contributions: Study concept and design: J.G. Laffey, G. Bellani, T. Pham and L. Brochard; acquisition of data: J.G. Laffey, G. Bellani, T. Pham and L. Brochard; analysis and interpretation of data: B.A. McNicholas, F. Madotto, E. Rezoagli, T. Pham, C.H. Masterson, S. Horie and J.G. Laffey; drafting of the manuscript: all authors; critical revision of the manuscript for important intellectual content: all authors; statistical analysis: F. Madotto, T. Pham and E. Rezoagli; obtained funding: G. Bellani and J.G. Laffey; study supervision: J.G. Laffey, G. Bellani and L. Brochard; approved the final draft of the manuscript: all authors.

Conflict of interest: B.A. McNicholas has nothing to disclose. F. Madotto has nothing to disclose. T. Pham has nothing to disclose. E. Rezoagli has nothing to disclose. C.H. Masterson has nothing to disclose. S. Horie has nothing to disclose. G. Bellani reports grants and personal fees from Draeger Medical, personal fees from Hamilton, Getinge and Dimar SRL, outside the submitted work. L. Brochard reports grants from Covidien, grants and nonfinancial support from Fisher Paykel, Air Liquide and General Electric, nonfinancial support from Philips and Sentec, outside the submitted work. J.G. Laffey has nothing to disclose.

\section{References}

1 Ayanian JZ, Epstein AM. Differences in the use of procedures between women and men hospitalized for coronary heart disease. N Engl J Med 1991; 325: 221-225.

2 Garland A, Olafson K, Ramsey CD, et al. Epidemiology of critically ill patients in intensive care units: a population-based observational study. Crit Care 2013; 17: R212.

3 Mnatzaganian G, Braitberg G, Hiller JE, et al. Sex differences in in-hospital mortality following a first acute myocardial infarction: symptomatology, delayed presentation, and hospital setting. BMC Cardiovasc Disord 2016; 16: 109.

4 Bierman AS, Brown AD, Levinton CM. Using decision trees for measuring gender equity in the timing of angiography in patients with acute coronary syndrome: a novel approach to equity analysis. Int J Equity Health 2015; 14: 155 .

5 Guha R, Boehme A, Demel SL, et al. Aggressiveness of care following intracerebral hemorrhage in women and men. Neurology 2017; 89: 349-354.

6 Valentin A, Jordan B, Lang T, et al. Gender-related differences in intensive care: a multiple-center cohort study of therapeutic interventions and outcome in critically ill patients. Crit Care Med 2003; 31: 1901-1907.

7 Bellani G, Laffey JG, Pham T, et al. Epidemiology, patterns of care, and mortality for patients with acute respiratory distress syndrome in intensive care units in 50 countries. JAMA 2016; 315: 788-800.

8 Rubenfeld GD, Cooper C, Carter G, et al. Barriers to providing lung-protective ventilation to patients with acute lung injury. Crit Care Med 2004; 32: 1289-1293.

9 Villar J, Blanco J, Añón JM, et al. The ALIEN study: incidence and outcome of acute respiratory distress syndrome in the era of lung protective ventilation. Intensive Care Med 2011; 37: 1932-1941.

10 Han S, Martin GS, Maloney JP, et al. Short women with severe sepsis-related acute lung injury receive lung protective ventilation less frequently: an observational cohort study. Crit Care 2011; 15: R262.

11 Walkey AJ, Wiener RS. Risk factors for underuse of lung-protective ventilation in acute lung injury. J Crit Care 2012; 27: 323.

12 Sasko B, Thiem U, Christ M, et al. Size matters: an observational study investigating estimated height as a reference size for calculating tidal volumes if low tidal volume ventilation is required. PLoS One 2018; 13: e0199917.

13 Nweze IC, Smith JW, Zhang B, et al. 17ß-Estradiol attenuates cytokine-induced nitric oxide production in rat hepatocyte. J Trauma Acute Care Surg 2012; 73: 408-412.

14 Bindl L, Buderus S, Dahlem P, et al. Gender-based differences in children with sepsis and ARDS: the ESPNIC ARDS Database Group. Intensive Care Med 2003; 29: 1770-1773.

15 Heffernan DS, Dossett LA, Lightfoot MA, et al. Gender and acute respiratory distress syndrome in critically injured adults: a prospective study. J Trauma 2011; 71: 878-883

16 Villar J, Martínez D, Mosteiro F, et al. Is overall mortality the right composite endpoint in clinical trials of acute respiratory distress syndrome? Crit Care Med 2018; 46: 892-899.

17 Lemos-Filho LB, Mikkelsen ME, Martin GS, et al. Sex, race, and the development of acute lung injury. Chest 2013; 143: 901-909.

18 Bellani G, Laffey JG, Pham T, et al. Noninvasive ventilation of patients with acute respiratory distress syndrome. Insights from the LUNG SAFE study. Am J Respir Crit Care Med 2017; 195: 67-77.

19 Laffey JG, Bellani G, Pham T, et al. Potentially modifiable factors contributing to outcome from acute respiratory distress syndrome: the LUNG SAFE study. Intensive Care Med 2016; 42: 1865-1876.

20 Madotto F, Pham T, Bellani G, et al. Resolved versus confirmed ARDS after 24 h: insights from the LUNG SAFE study. Intensive Care Med 2018; 44: 564-577.

21 Laffey JG, Madotto F, Bellani G, et al. Geo-economic variations in epidemiology, patterns of care, and outcomes in patients with acute respiratory distress syndrome: insights from the LUNG SAFE prospective cohort study. Lancet Respir Med 2017; 5: 627-638.

22 Bos MM, Verburg IW, Dumaij I, et al. Intensive care admission of cancer patients: a comparative analysis. Cancer Med 2015; 4: 966-976.

23 Walkey AJ, Wiener RS. Risk factors for underuse of lung-protective ventilation in acute lung injury. J Crit Care 2012; 27: 323.

24 Brower RG, Matthay MA, Morris A, et al. Ventilation with lower tidal volumes as compared with traditional tidal volumes for acute lung injury and the acute respiratory distress syndrome. N Engl J Med 2000; 342: 1301-1308.

25 Mikkelsen ME, Dedhiya PM, Kalhan R, et al. Potential reasons why physicians underuse lung-protective ventilation: a retrospective cohort study using physician documentation. Respir Care 2008; 53: 455-461.

26 Needham DM, Yang T, Dinglas VD, et al. Timing of low tidal volume ventilation and intensive care unit mortality in acute respiratory distress syndrome. A prospective cohort study. Am J Respir Crit Care Med 2015; 191: 177-185.

27 Fernandez-Bustamante A, Wood CL, Tran ZV, et al. Intraoperative ventilation: incidence and risk factors for receiving large tidal volumes during general anesthesia. BMC Anesthesiol 2011; 11: 22. 
28 Fowler RA, Sabur N, Li P, et al. Sex-and age-based differences in the delivery and outcomes of critical care. CMAJ 2007; 177: 1513-1519.

29 Mahmood K, Eldeirawi K, Wahidi MM. Association of gender with outcomes in critically ill patients. Crit Care 2012; 16: R92.

30 Chotirmall SH, Greene CM, Oglesby IK, et al. 17Beta-estradiol inhibits IL-8 in cystic fibrosis by up-regulating secretory leucoprotease inhibitor. Am J Respir Crit Care Med 2010; 182: 62-72.

31 Bastarache JA, Ong T, Matthay MA, et al. Alveolar fluid clearance is faster in women with acute lung injury compared to men. J Crit Care 2011; 26: 249-256.

32 El-Haddad $\mathrm{H}$, Jang $\mathrm{H}$, Chen $\mathrm{W}$, et al. The effect of demographics and patient location on the outcome of patients with acute respiratory distress syndrome. Ann Thorac Med 2017; 12: 17-24.

33 Kollef MH, O'Brien JD, Silver P. The impact of gender on outcome from mechanical ventilation. Chest 1997; 111: $434-441$.

34 Sinuff T, Cook DJ, Rocker GM, et al. DNR directives are established early in mechanically ventilated intensive care unit patients. Can J Anaesth 2004; 51: 1034-1041.

35 Skjaker SA, Hoel H, Dahl V, et al. Factors associated with life-sustaining treatment restriction in a general intensive care unit. PLoS One 2017; 12: e0181312. 